\title{
Electron-phonon heat transfer in giant vortex states
}

\author{
A. V. Samokhvalov, ${ }^{1,2}$ I. A. Shereshevskii, ${ }^{1}$ N. K. Vdovicheva, ${ }^{1}$ M. Taupin $\odot,{ }^{3}$ I. M. Khaymovich $\odot,{ }^{1,4}$ and A. S. Mel'nikov ${ }^{1,2}$ \\ ${ }^{1}$ Institute for Physics of Microstructures, Russian Academy of Sciences, 603950 Nizhny Novgorod, Russia \\ ${ }^{2}$ Lobachevsky State University of Nizhni Novgorod, 23 Prospekt Gagarina, 603950 Nizhni Novgorod, Russia \\ ${ }^{3}$ Institute of Solid State Physics, Vienna University of Technology, Wiedner Hauptstrasse 8-10, 1040 Vienna, Austria \\ ${ }^{4}$ Max Planck Institute for the Physics of Complex Systems, Nöthnitzer Str. 38, 01187 Dresden
}

(Received 30 November 2021; accepted 21 January 2022; published 31 January 2022)

\begin{abstract}
We examine energy relaxation of nonequilibrium quasiparticles (QPs) in different vortex configurations in "dirty" $s$-wave superconductors (SCs). The heat flow from the electronic subsystem to phonons in a mesoscopic SC disk with a radius of the order of several coherence lengths is calculated both in the Meissner and in the giant vortex states using the Usadel approach. The recombination process is shown to be strongly affected by interplay of the subgap states, located in the vortex core and in the region at the sample edge where the spectral gap $E_{\mathrm{g}}$ is reduced by the Meissner currents. In order to uncover the physical origin of the results, we develop a semiquantitative analytical approximation based on the combination of homogeneous solutions of Usadel equations in Meissner and vortex states of a mesoscopic SC disk and analytically calculate the corresponding spatially resolved electron-phonon heat rates. Our approach provides important information about nonequilibrium QPs cooling by the magnetic field-induced traps in various mesoscopic SC devices.
\end{abstract}

DOI: 10.1103/PhysRevB.105.024522

\section{INTRODUCTION}

The progress in cryogenic superconducting (SC) devices and technologies, such as electronic refrigerators and thermometers [1], radiation detectors [2,3], and qubit systems for quantum information processing [4,5], requires an improved understanding of the quasiparticle (QP) thermalization mechanisms. Such mesoscopic devices with nanoscale dimensions operating at subkelvin temperatures are easily driven out of equilibrium via processes involving electromagnetic-field absorption, hot QP injection, or the operational drive. As a result, there is a significant concentration of nonequilibrium QPs present in a driven superconductor even for temperatures far away from the drive location well below the temperature of SC transition [6]. Excess QPs destroy coherence of qubit systems [7-9], decrease the quality factor of SC resonators $[10,11]$, reduce the efficiency of cooling in electronic refrigerators $[12,13]$, and result in the excess current in single electron [14-16] and heat quantum [17] turnstiles. The energy-relaxation rate of the nonequilibrium QPs is also known to affect the characteristics of detectors of electromagnetic radiation $[6,18]$. Thus the overheating of QPs or unwanted population in general appears to be a major factor limiting the performance of low-temperature SC devices. This is why the study of the mechanisms of nonequilibrium

Published by the American Physical Society under the terms of the Creative Commons Attribution 4.0 International license. Further distribution of this work must maintain attribution to the author(s) and the published article's title, journal citation, and DOI. Open access publication funded by the Max Planck Society.
QPs relaxation in SCs of mesoscopic dimensions seems to be rather important (see, e.g., Ref. [19] for review). Typically, the presence of excess QPs is characterized by an effective electron temperature $T$, which exceeds the temperature of the phonon bath $T_{\mathrm{ph}}$. This approximation is relevant for various SC devices at low temperatures (including the above-mentioned ones), when the inelastic electron-electron scattering time $\tau_{e e}$ is small compared to the operation times, while the electron-phonon (e-ph) relaxation time is large with respect to the operation times.

In order to suppress QP poisoning and to prevent overheating, density of nonequilibrium QPs in a device should be reduced. Simple lowering the phonon-bath temperature $T_{\mathrm{ph}}$ often is not sufficient, and to remove QPs in SC devices additional QP traps should be used: Either normal-metal films connected to the SC [20-22], or Andreev bound states in weak links [23], or regions with reduced or destroyed SC gap [24-27]. In this case, QPs are trapped by the region with no energy gap (or suppressed gap) away from the active region. One of the possible types of QPs traps can be formed by regions with the suppressed SC gap that appear in the Meissner and vortex states and can be successfully controlled by the external magnetic field [6,28-35]. This method has a number of advantages due to using of the same material as the rest of the device, since perfect matching of different parts of the device without barriers or interface potentials is provided. Besides, the magnetic field allows to tune the trap controllably. The controllable use of such traps in various applications mentioned above assumes, certainly, understanding of their cooling capacities.

The hot electrons dissipate the heat typically via the interaction with the phonons [36-38]. In three-dimensional normal metals of volume $\mathcal{V}$ (when the mean-free path $\ell$ is small 
compared to all other length scales), the heat flow $\dot{Q}_{N}$ related to the e-ph relaxation is determined by the well-known relation

$$
\dot{Q}_{N}=\Sigma \mathcal{V}\left(T^{5}-T_{\mathrm{ph}}^{5}\right),
$$

where $\Sigma$ is the material constant for e-ph coupling [1]. QP recombination in a bulk SC with an $s$-wave gap via coupling to the phonons has been widely studied [38-42]. The hard energy-gap $E_{\mathrm{g}}$ in the QP spectrum of the $\mathrm{SC}$ is known to suppress the recombination of the hot QPs making the relaxation rate $\nu_{e-p h}$ to be exponentially slow $\left[\sim \exp \left(-E_{\mathrm{g}} / k_{B} T\right)\right]$ because QPs need to possess an energy exceeding the gap $E_{\mathrm{g}}$ in order to recombine. The e-ph relaxation remains extremely slow even in the presence of Abrikosov vortices and subgap excitations localized in the vortex core in clean SCs at ultralow temperatures [43]. The resulting relaxation rate enters the rate equations for the concentration of QPs and phonons which allow to get the full description of the nonequilibrium processes in SC devices [44]. The e-ph relaxation in mesoscopic SC samples and SC point contacts looks rather different compared to dynamics of bulk QPs [45-48]. The simplified model of QP dynamics and electron cooling both in the Meissner and in the vortex states of a mesoscopic SC was proposed recently in Ref. [33] to explain experimental measurements of the characteristics of nonequilibrium QP distributions in a mesoscopic SC island in a single-electron transistor setup with normal metal leads; however, that model worked well only for the Meissner and single-quantum vortex states.

The main goal of our work is to overcome this limitation by developing a quantitative description of the electron-phonon heat transfer processes in a diffusive mesoscopic SC disk of the size comparable to several SC coherence lengths $\xi_{0}$ placed in an external magnetic field oriented perpendicular to the plane of the disk. In such nanoscale samples, theory predicts the existence of rather exotic vortex configurations so-called "giant" (or multiquantum) vortices in the center of a circular-formed sample (disk) [49,50]. These exotic vortex states are formed due to the confinement effect of the screening supercurrents and have been observed by a variety of experimental methods [49-54], including scanning tunneling microscopy/spectroscopy studies [55-60]. As has been shown in several works, the realization of giant vortices is not limited to the samples of a circular form [57]. Indeed, in certain ranges of magnetic field giant vortices have been observed in square-shaped samples [61] and predicted in the sample of different shapes. Depending on the particular sample geometry and the details of the hysteretic magnetization procedure, the giant vortex states can decay in vortex molecules of different configurations resulting, of course, in some peculiarities in the field dependencies of free energy and magnetization [55,61]. In our work we focus on a circular sample shape and central position of a vortex in order to simplify consideration.

The electronic structure of mesoscopic SCs is known to be sensitive to the applied magnetic field, related vorticity, and vortex configurations [62-68]. The overall spectral characteristics and local density of states (LDOS) of the mesoscopic disk were shown to be strongly affected by an interplay of the subgap states, located in the vortex core and in the region

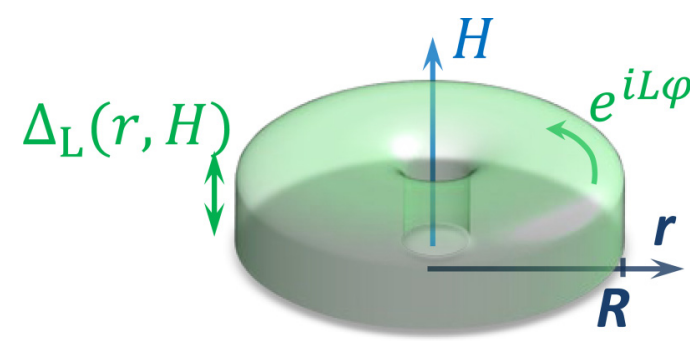

FIG. 1. Schematic picture of the spatial order parameter distribution $\Delta_{L}(H)$ (shown by semitransparent green color) in the SC disk of the radius $R$ with the giant $L$-quantum vortex, Eq. (2), in the applied perpendicular magnetic field $H$.

at the sample edge where the spectral gap $E_{\mathrm{g}}$ is reduced by Meissner currents [69]. Keeping in mind the high sensitivity of e-ph relaxation to the LDOSs [33], one can expect a strong dependence of the total QPs heat flow and the relaxation rate $v_{e-p h}$ in the mesoscopic disk on the applied magnetic field and related vorticity. Note that both the giant vortex cores and the region near the sample edge with the flowing Meissner screening currents can be clearly viewed as QP traps created by the applied magnetic field. It is the joint effect of contributions from the core and edge traps, which determines the total QPs heat flow in a mesoscopic sample.

The paper is organized as follows. In Sec. II we briefly discuss the basic equations. In Sec. III we calculate the SC critical temperature $T_{\mathrm{c}}$ and study the switching between states with different vorticity $L$ while sweeping the magnetic field. In Sec. IV we calculate numerically the e-ph heat flow in a mesoscopic SC disk with a giant vortex. In Sec. V we propose a simplified analytical model to describe the e-ph heat flow and check its applicability. We summarize our results in Sec. VI.

\section{MODEL AND BASIC EQUATIONS}

To set the stage, here we describe the considered system and main relevant equations and approximations. The section contains the standard notations and literature results; therefore a reader, acquainted with this formalism and interested in the original result, can directly jump to Sec. III.

The system in the focus is a thin mesoscopic SC disk of a radius $R$ comparable to several SC coherence lengths $\xi$ at a finite temperature $T$. We concentrate on more experimentally relevant $s$-wave SCs in the dirty limit where the mean-free path $\ell$ is the smallest length scale in the system. In terms of the elastic electron scattering rate $\tau^{-1}$ and the bare SC transition temperature $T_{\mathrm{cs}}$, the above dirty limit condition reads as follows: $k_{B} T_{\mathrm{cs}} \tau / \hbar \ll 1$. In order to generate vortices that trap QPs in their cores, an external magnetic field $\mathbf{H}=H \mathbf{z}_{0}$, perpendicular to the SC disk plane, is applied (Fig. 1). We focus on the experimentally relevant situation of a small disk thickness $d$ and disregard the magnetic field, induced by Meissner supercurrents in the disk. This approximation holds as soon as $d$ is small compared to the London penetration depth $\lambda$ in such a way that the effective magnetic field penetration depth $\Lambda=\lambda^{2} / d$ is large compared to the disk radius $\Lambda \gg R$. This allows us to take into account only the external field, i.e., 
$\operatorname{rot} \mathbf{A}=\mathbf{B} \equiv \mathbf{H}$. In addition, due to smallness of the inelastic electron-electron scattering time $\tau_{e e}$ with respect to the e-ph one in experimentally relevant setups, we assume that the QP energy distribution in a SC disk is the quasiequilibrium Fermi distribution $f_{T}(E)=\left[e^{E / k_{B} T}+1\right]^{-1}$, characterized by a certain (electronic) temperature $T \ll T_{\mathrm{cs}}$ which may differ from the bath temperature $T_{\mathrm{ph}}$. This approximation is appropriate for various low-temperature SC devices (see Introduction); therefore we take the liberty to consider our open electronic subsystem using the steady-state heat flow equations with a well-defined electronic temperature. In this regime, the normal $(\mathcal{G})$ and anomalous $(\mathcal{F})$ quasiclassical Green's functions obey the Usadel equations [70], which are valid for all temperatures and for distances exceeding the mean-free path $\ell$. We concentrate on the cylindrically symmetric case, introduce the coordinates $(r, \varphi, z)$ as it is shown in Fig. 1, and look for the homogeneous solution along the $z$-axis solutions characterized by a certain integer angular momentum $L$ (called a vorticity),

$$
\Delta(\mathbf{r})=\Delta_{L}(r) \mathrm{e}^{i L \varphi},
$$

which describes the axisymmetric multiquantum vortex states with the vortex core located at the center of the disk, $r=0$. The vorticity $L$ coincides with the angular momentum of the anomalous Green's function $\mathcal{F}$. In the standard trigonometrical parametrization the quasiclassical Green's functions for $L$-th orbital mode can be encoded in the pairing angle $\theta_{L}(r)$ as

$$
\mathcal{G}=\cos \theta_{L}, \quad \mathcal{F}=\sin \theta_{L} \mathrm{e}^{i L \varphi}, \quad \mathcal{F}^{\dagger}=\sin \theta_{L} \mathrm{e}^{-i L \varphi} .
$$

The pairing angle $\theta_{L}$ obeys the following equation (see, e.g., Ref. [71])

$$
\begin{aligned}
-\frac{\hbar D}{2} \nabla_{r}^{2} \theta_{L} & +\left[\omega_{n}+\Gamma_{L}(r, H) \cos \theta_{L}\right] \sin \theta_{L} \\
& =\Delta_{L}(r) \cos \theta_{L},
\end{aligned}
$$

where the inhomogeneous depairing parameter

$$
\Gamma_{L}(r, H)=\hbar v_{L}^{2} / 2 D
$$

is expressed through the superfluid velocity $\mathbf{v}_{s}=\left(0, v_{L}, 0\right)$

$$
v_{L}=D\left(\frac{L}{r}-\frac{\pi H}{\Phi_{0}} r\right)
$$

and depends on the external magnetic field $H$. Here $\omega_{n}=$ $\pi T(2 n+1)$ is the Matsubara frequency at temperature $T$, $D=v_{\mathrm{F}} l / 3$ is the diffusion coefficient, and $\Phi_{0}=\pi \hbar c / e$ is the flux quantum. The relevant length scale in the Usadel equations is given by the SC coherence length $\xi_{0}=\sqrt{\hbar D / 2 \Delta_{0}}$, where $\Delta_{0}$ is the SC gap at zero temperature. Further, we treat only positive $\omega_{n}$ values due to the symmetry of Usadel equations and $\mathcal{F}$ being an even function of $\omega_{n}, \mathcal{F}\left(\mathbf{r},-\omega_{n}\right)=$ $\mathcal{F}\left(\mathbf{r}, \omega_{n}\right)$. The singlet pairing potential $\Delta_{L}(r)$ in Usadel equations (3) is determined self-consistently by the equation

$$
\frac{\Delta_{L}(r)}{g}-2 \pi T \sum_{n \geqslant 0} \sin \theta_{L}=0,
$$

where the pairing parameter $g$ fixes the bare critical temperature $T_{\mathrm{cs}}$ as

$$
\frac{1}{g}=\sum_{n=0}^{\Omega_{\mathrm{D}} /\left(2 \pi T_{\mathrm{cs}}\right)} \frac{1}{n+1 / 2} \simeq \ln \left[\frac{\Omega_{\mathrm{D}}}{2 \pi T_{\mathrm{cs}}}\right]+2 \ln 2+\gamma
$$

Here $\Omega_{\mathrm{D}}$ is the Debye frequency and $\gamma \simeq 0.5772$ is the EulerMascheroni constant. Equations (3) and (6) in the disk bulk should be accompanied by the boundary conditions at the disk edge $r=R$ for the order parameter $\Delta_{L}$ and the pairing angle $\theta_{L}$ :

$$
\left.\frac{d \Delta_{L}}{d r}\right|_{R}=0,\left.\quad \frac{d \theta_{L}}{d r}\right|_{R}=0 .
$$

Both the Usadel (3) and the self-consistency (6) equations can be obtained by variation of the free energy functional

$$
F_{L}=2 \pi N_{0} d\left(\pi T \sum_{\omega_{n}<\Omega_{\mathrm{D}}} \int_{0}^{R} r d r\left\{\hbar D\left(\frac{\partial \theta_{L}}{\partial r}\right)^{2}+2 \Gamma_{L} \sin ^{2} \theta_{L}-4 \omega_{n} \cos \theta_{L}-4 \Delta_{L} \sin \theta_{L}\right\}+\frac{1}{g} \int_{0}^{R} r d r \Delta_{L}^{2}\right),
$$

with $N_{0}$ representing the DOSs at the Fermi level for a spin projection.

The power absorbed in the disk is associated with the heat transferred to phonons emitted by thermal QPs. In a general inhomogeneous case, the electron-phonon heat flow $\dot{Q}_{L}(r)$ across the central part of the disk of radius $r \leqslant R$ into the phonon bath for the orbital mode $L$ is given by the expressions [40,41]

$$
\dot{Q}_{L}(r)=2 \pi d \int_{0}^{r} d r^{\prime} r^{\prime} \mathcal{P}_{L}\left(r^{\prime}\right), \quad \mathcal{P}_{L}(r)=\int_{0}^{\infty} \frac{\Sigma\left[n_{T}(\epsilon)-n_{T_{\mathrm{ph}}}(\epsilon)\right]}{24 \zeta(5) k_{B}^{5}} \epsilon^{3} d \epsilon \int_{-\infty}^{\infty} M_{L}(r, E, \epsilon)\left[f_{T}(E)-f_{T}(E+\epsilon)\right] d E .
$$

Here $\zeta(s)$ is the Riemann zeta function, $k_{B}$ is the Boltzmann constant, and $n_{T}(\epsilon)=\left[\exp \left(\epsilon / k_{B} T\right)-1\right]^{-1}$ is the Bose function with a temperature $T$. The kernel of the integrals (10)

$$
M_{L}(r, E, \epsilon)=N_{L}(r, E) N_{L}(r, E+\epsilon)-B_{L}(r, E) B_{L}(r, E+\epsilon),
$$

with the LDOS $N_{L}(r, E)$ and SC correlations $B_{L}(r, E)$

$$
N_{L}(r, E)=\left.\operatorname{Re}\left[\cos \theta_{L}(r)\right]\right|_{\omega_{n}=-i E}, \quad B_{L}(r, E)=\left.\operatorname{Im}\left[\sin \theta_{L}(r)\right]\right|_{\omega_{n}=-i E},
$$

depending on the radial coordinate $r$ due to the spatial dependence of the normal $\left(\sim \cos \theta_{L}\right)$ and anomalous $\left(\sim \sin \theta_{L}\right)$ Green's functions in the disk. 


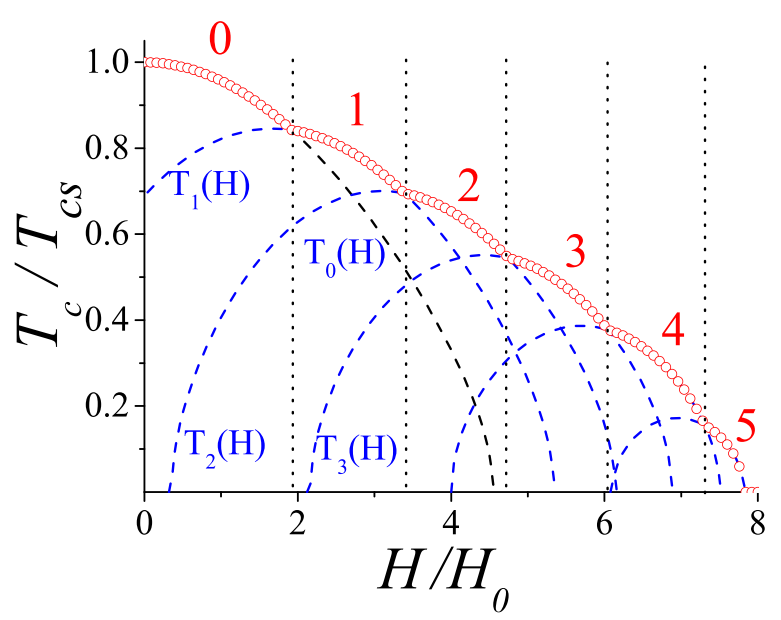

FIG. 2. Schematic dependence of the critical temperature $T_{L}$ ( $L=1 \div 5$ ) (dashed blue lines) and $T_{\mathrm{c}}$ (red symbols) on the external magnetic field $H$. The vorticity $L$ is denoted by the numbers near the curves. The values of the magnetic field $H=H_{L}$ corresponding to the switching of the orbital modes between $L$ and $L+1$ are shown by the dotted vertical lines.

To calculate $M_{L}(r, E, \epsilon)$ we have to solve the Usadel equation (3) with the inhomogeneous depairing parameter $\Gamma_{L}(r, H)(4),(5)$ for $\omega_{n}=-i E$ in the presence of the $L$-quantum vortex in the disk center. The solution of the Usadel equation gives us the LDOS $N_{L}(r, E)$ and the spectral gap value $E_{\mathrm{g}}$ governing the contribution to the thermal relaxation mechanisms (see, e.g., Refs. [33,34]). From the experimental point of view, LDOS $N_{L}(r, E)$ can be measured directly via the local differential conductance (e.g., in the scanning tunneling spectroscopy setting):

$$
\begin{aligned}
G_{L}(r, V) & =\frac{d I / d V}{(d I / d V)_{N}} \\
& =\int_{-\infty}^{\infty} d E \frac{N_{L}(r, E)}{N_{0}} \frac{\partial f_{T}(E-e V)}{\partial V} .
\end{aligned}
$$

Here $V$ is the applied bias voltage; $(d I / d V)_{N}$ is the conductance of the normal metal junction.

\section{GIANT VORTEX STATES IN MESOSCOPIC DISK}

The phase boundary $T_{\mathrm{c}}(H)$ of the mesoscopic SC disk is known to exhibit an oscillatory behavior similar to the well-known Little-Parks oscillations [72,73], caused by transitions between states with different angular momenta $L$. Here $T_{\mathrm{c}}(0)=T_{\mathrm{cs}}$. Figure 2 shows a typical dependence of the critical temperature,

$$
T_{\mathrm{c}}(H)=\max _{L}\left\{T_{L}(H)\right\},
$$

on the external magnetic field $H$, affected by these transitions. The values of the normalized flux $\phi_{L}=\pi H_{L} R^{2} / \Phi_{0}=H_{L} / H_{0}$ through the disk of a radius $R$, where the switching of the orbital modes $L \rightleftarrows L+1$ takes place, do not depend on the disk radius $R$ and can be found using Eqs. (3) and (6) linearized in the anomalous Green's function $\left(\cos \theta_{L} \simeq 1, \sin \theta_{L} \simeq \theta_{L}\right.$ ) [69] or within the Ginzburg-Landau formalism [74] based on the free energy (9). Note that this energy-based approach (9) does

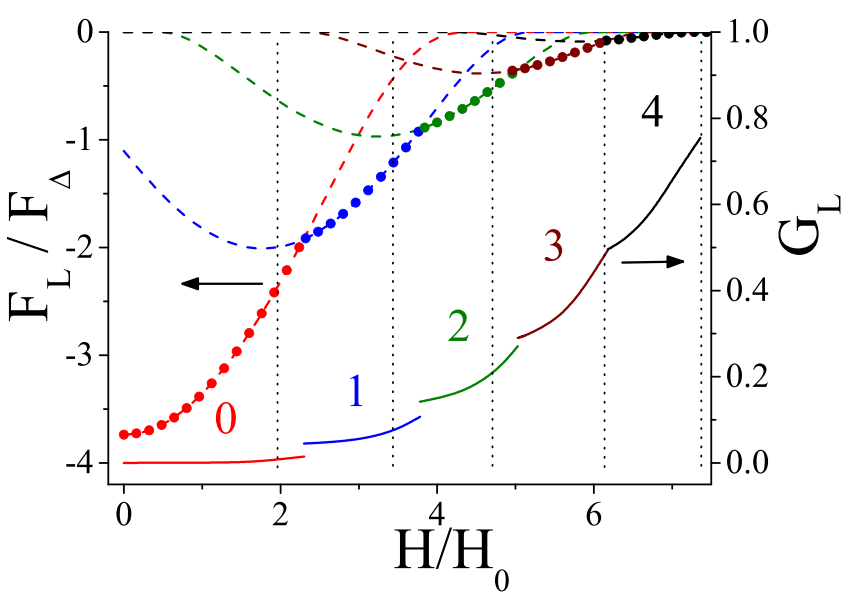

FIG. 3. The magnetic flux $\phi=H / H_{0}$ dependence of the normalized ZBC $G_{L}(0, R)(13)$ at the edge of the SC disk (solid lines) and the free energy $F_{L}(9)$ (symbol $\bullet$ ) for the temperatures $T=0.1 T_{\text {cs }}$. The disk radius is $R=4 \xi_{0}$; the SC coupling constant is $g=0.18$. The dependence $F_{L}(\phi)$ for fixed vorticity $\mathrm{L}=0 \div 4$ is shown by the dashed lines. Like in Fig. 2, the corresponding values of vorticity $L$ are denoted by the numbers near the curves, while the values of the flux $H_{L} / H_{0}=\phi_{L}$ corresponding to the switching of the orbital modes between $L$ and $L+1$, with different critical temperatures $T_{\mathrm{c}}$, are shown by the dotted vertical lines. Here the free energy is normalized by $F_{\Delta}=\pi \hbar D N_{0} d \Delta_{0}$.

not take into account possible hysteresis in the orbital-mode switching caused by the barriers between different free-energy minima. In order to analyze the transitions between different vortex states far from the phase transition line $T \ll T_{\mathrm{c}}(H)$ we need to use the nonlinear Usadel theory [(3)-(8)]. The Usadel equations have been solved numerically for different vorticities $L$ which allowed us to calculate and compare the values of the free energy $F_{L}(9)$. Figure 3 shows the magnetic field dependence of the free energy (9) and the zero-bias conductance (ZBC) $G_{L}(R, 0)(13)$ at the Fermi level for a small disk radius $R=4 \xi_{0}$ and the temperature $T=0.1 T_{\text {cs }}$. The curves illustrate the switching between the states with different vorticities $L=0 \div 4$, which is similar to the LittleParks-like switching of the critical temperature $T_{\mathrm{c}}(H)$ shown in Fig. 2.

Sequential entries of vortices produce a set of branches $F_{L}$ with different vorticity $L$ on the $F(H)$ and $d I / d V(H)$ curves. The transitions between different vortex states are accompanied by an abrupt change in the ZBC at the disk edge, which is attributed to the entry/exit of a vortex while sweeping the magnetic field. Note that the field values $H_{s L}$ at which the jumps in vorticity $(L-1 \rightarrow L)$ occur at low temperature are always larger than the values $H_{L}$ found from the calculations of the critical temperature behavior $T_{\mathrm{c}}(H)$. For a fixed disk radius $R$ the direction of jumps (upward/downward) in the dependence of $\mathrm{ZBC} G_{L}(R, 0)$ vs magnetic field depends on temperature $T$ and reflects a crossover between the edgedominated and core-dominated regimes in the magnetic field dependence of the tunneling conductance (see Ref. [69] for details). Figure 4 illustrates the evolution of the spatially resolved LDOS $N_{L}(r, E)$ vs energy $E$ for values of the magnetic field $H=H_{s 1}, H_{s 2}$ corresponding to the vorticity switching. 

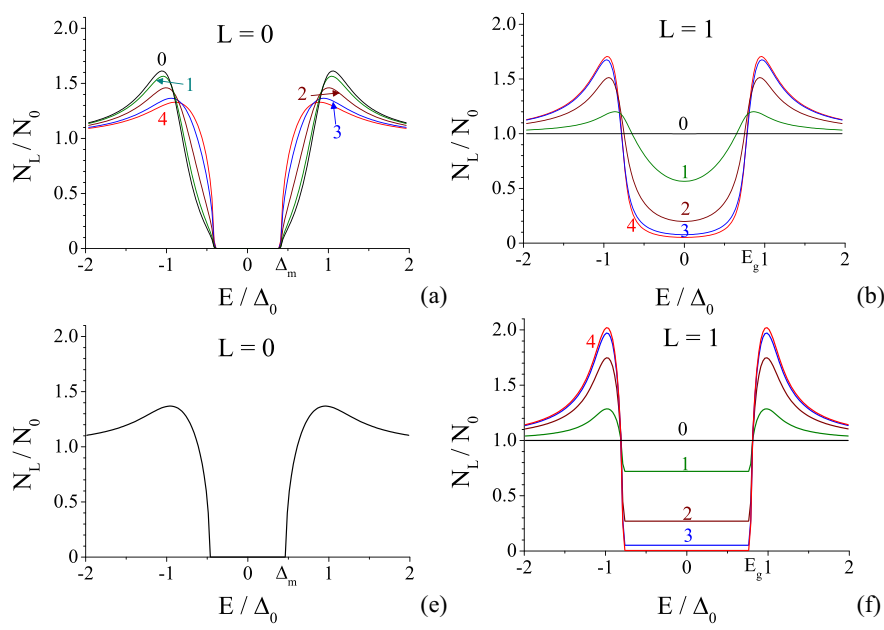

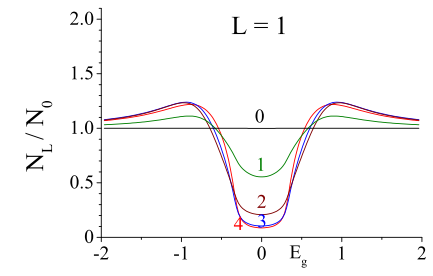

(b)

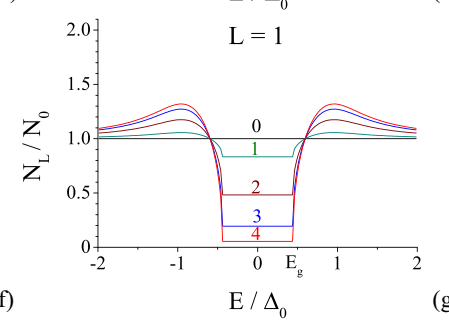

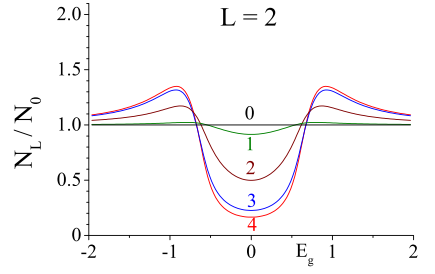

(c)

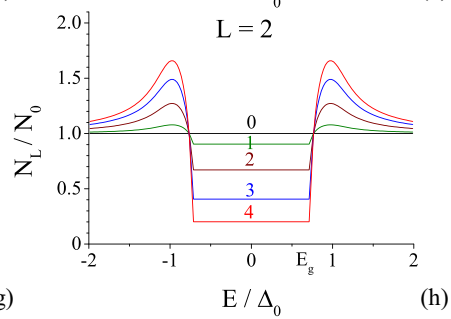

FIG. 4. Comparison of the exact (12) (panels a-d) and approximate (14) (panels e-h) LDOS $N_{L}(r, E$ ) vs energy $E$ for several distances $r$ from the vortex core, vorticities $L$, and magnetic fields $H$. The SC disk radius $R=4 \xi_{0}$ and the temperature $T=0.1 T_{\mathrm{cs}}$ are fixed for all panels. The magnetic field values $H$ are (a, b, e, f) $H=H_{s 1}=2.24 H_{0}$; (c, d, g, h) $H=H_{s 2}=3.84 H_{0}$. The distances $r$ (in the units of $\xi_{0}$ ) are shown by the numbers of the corresponding colors near the curves. The soft minigap at the edge $E_{\mathrm{g}}$ in $(\mathrm{b}-\mathrm{d})$ is assumed to correspond to the maximal slope of the energy dependence of the $\operatorname{LDOS} d N_{L}(R, E) / d E$.

In the Meissner state $(L=0)$, the hard minigap $\Delta_{\mathrm{m}}$ in the spectrum survives and $N_{L=0}\left(r, E<\Delta_{\mathrm{m}}\right)=0$ until the first vortex entry; Fig. 4(a). In vortex states $(L \geqslant 1)$, Figs. 4(b) and $4(\mathrm{~d})$, the $\operatorname{DOS} N_{L}(0, E)$ is equal to the normal-metal electronic DOS at the Fermi level $N_{0}$, indicating a full suppression of the spectral gap in the disk center due to the vortex entry. At the same time, at the edge of the disk, the SC survives, but the gap becomes soft, $0<N_{L}(R, E)<N_{0}$. The soft minigap at the edge $E_{\mathrm{g}}$ is assumed to correspond to the maximal slope of the energy dependence of the LDOS $d N_{L}(R, E) / d E$. Figures 4(a) and 4(b) illustrate the switching between the states with hard and soft gaps at $H=H_{s 1} \simeq 2.24 H_{0}$ when the Meissner state $(L=0)$ transforms to the single-vortex state $(L=1)$. At fixed vorticity $L=1$ as the magnetic field increases, the LDOS $N_{L}(r, E)$ smoothly evolves with the simultaneous decrease of the soft minigap at the disk edge $E_{\mathrm{g}}$; Figs. 4(b) and 4(c). This smooth evolution of the LDOS is interrupted at $H=H_{s 2} \simeq 3.84 H_{0}$ by a next vortex entry which restores the SC near the disk edge and results in an increase of the soft minigap $E_{\mathrm{g}}$; Figs. 4(c) and 4(d). Certainly these drastic changes in the LDOS $N_{L}(r, E)$ and the spectral gap value $E_{\mathrm{g}}$ directly impact the thermal relaxation mechanisms and manifest themselves in peculiarities of the magnetic field dependence of the electron-phonon heat flow $\dot{Q}$, which we consider further.

\section{ELECTRON-PHONON HEAT FLOW IN GIANT VORTEX CONFIGURATIONS}

As a next step we calculate the thermal relaxation rate in the SC disk assuming the electronic temperature $T$ to be much larger than the bath (phonon) temperature $T_{\mathrm{ph}}$, thus neglecting for simplicity all the exponential terms like $e^{-E_{g} / k_{B} T_{\mathrm{ph}}}$. The filled circles in Fig. 5 show the magnetic field dependence of the total e-ph heat flow $\dot{Q}$ vs the applied magnetic field $H$ for a small disk. Here and further we focus on the disk radius $R=4 \xi_{0}$ and the temperature $T=0.1 T_{\mathrm{cs}}$ (if not mentioned otherwise). The total $\dot{Q}(R)$ curve consists of several separate branches $\dot{Q}_{L}(10)$ corresponding to the states with different vorticity $L=0 \div 4$. The transitions between different vortex states are visualized by abrupt changes (or jumps) $\triangle \dot{Q}$ in the heat flow at $H=H_{s L}$, where switching of the orbital modes $L-1 \rightleftarrows L$ takes place. The smooth growth of the e-ph heat flow $\dot{Q}_{L}$ occurs while sweeping the magnetic field up within the branch $L, H_{s L}<H<H_{s(L+1)}$ due to a decrease in the value of the spectral gap (soft or hard) and an increase in the subgap LDOS at the disk edge [69]. The especially strong

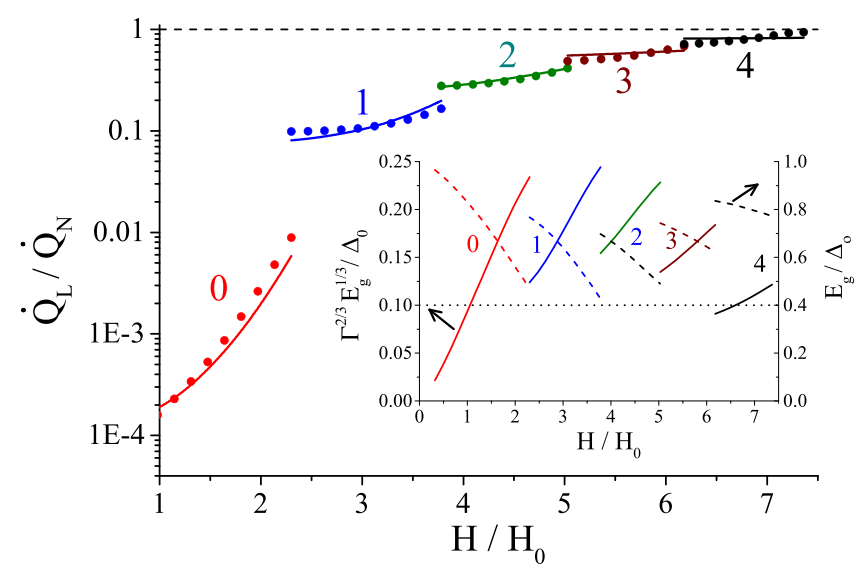

FIG. 5. The dependence of the e-ph heat flow $\dot{Q}_{L}(R)(10)$ on the magnetic flux $\phi=H / H_{0}$ across the SC disk of the radius $R=4 \xi_{0}$ for temperatures $T=0.1 T_{\text {cs }}$ (filled circles). The solid lines show the results of fitting $\dot{\bar{Q}}_{L}$ (26), (27) in homogeneous approximation described in Sec. V. The inset shows the dependence of the soft gap $\bar{E}_{g}$ (solid lines) and the value $\bar{\Gamma}^{2 / 3} \bar{E}_{g}^{1 / 3}$ (dashed lines) determined by the relations (17) on the magnetic flux $\phi$. The dotted line shows the value of the temperature $T$. The numbers near the curves denote the corresponding values of vorticity $L=0 \div 4 .\left(\dot{Q}_{N}=\Sigma \mathcal{V} T^{5}\right.$, $\left.\mathcal{V}=\pi R^{2} d\right)$. 

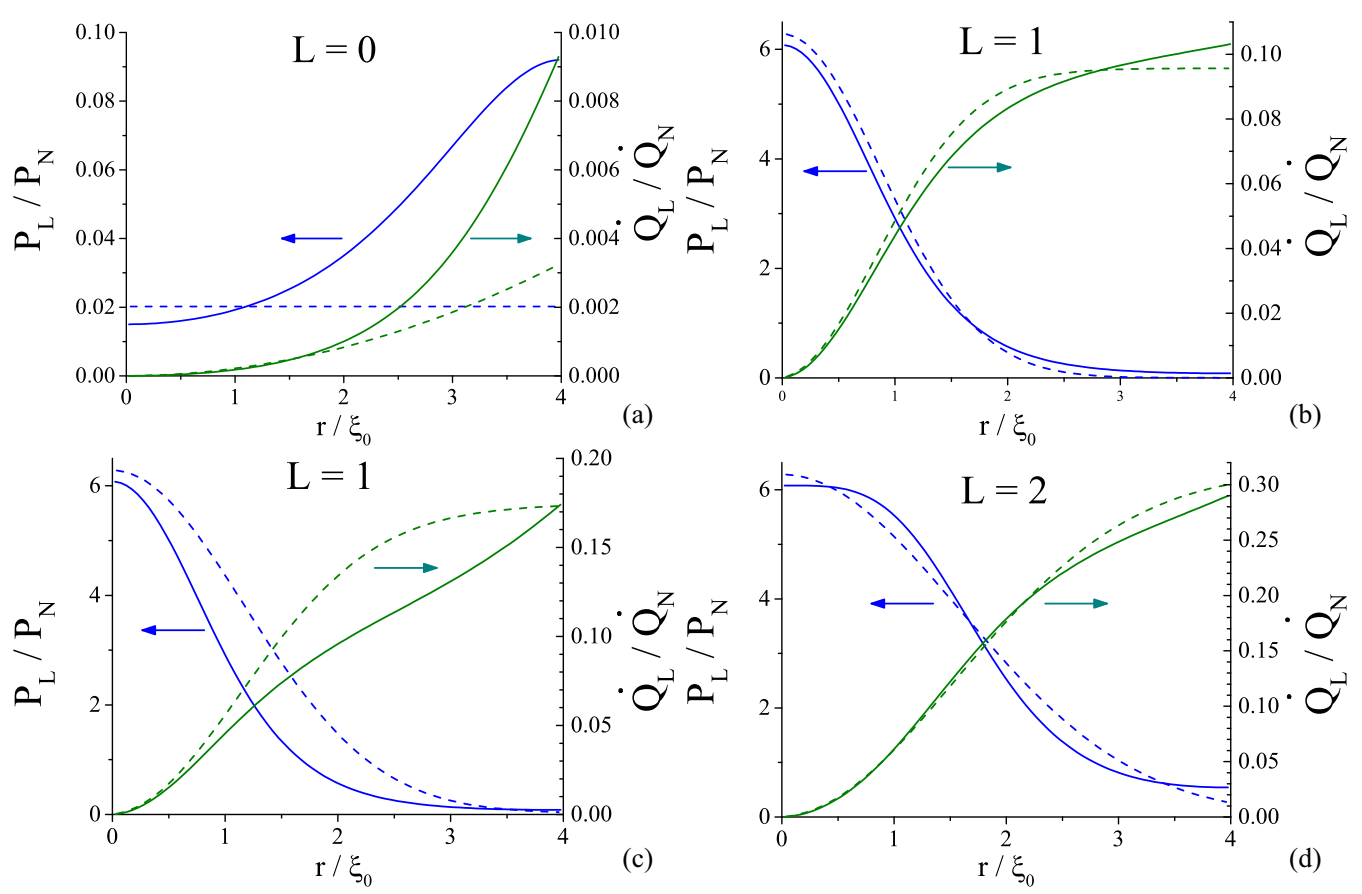

FIG. 6. The spatially resolved heat flow $\dot{Q}_{L}(r)$ and the density of the e-ph heat flow $P_{L}(r)$ (10) (solid lines) for several values of magnetic field $H$ : (a, b) $H=H_{s 1}=2.24 H_{0}$; (c, d) $H=H_{s 2}=3.84 H_{0}$. The dashed lines show the results of fitting $\dot{Q}_{L}(r)(26),(27)$ for $Z_{\mathcal{D}_{L}}(r)=\mathrm{e}^{-r^{2} / \mathcal{D}_{L}^{2}}$ $(L \geqslant 1)$ described in Sec. V: $D_{1}=1.6 \xi_{0}, D_{2}=2.7 \xi_{0} .\left(R=4 \xi_{0}, T=0.1 T_{\text {cs }}, \dot{Q}_{N}=\Sigma \mathcal{V} T^{5}, P_{N}=\Sigma T^{5}, \mathcal{V}=\pi R^{2} d\right)$.

growth of $\dot{Q}_{L}$ appears in the Meissner state $\left(\dot{Q}_{0}\left(H_{s 1}\right) / \dot{Q}_{0}(0) \sim\right.$ $10^{2}$ ) owing to essential suppression of the hard minigap $\Delta_{m}$ in the spectrum by the screening currents; Fig. 4(a).

To compare the contributions to the total e-ph heat flow $\dot{Q}(R)$ (10) from the vortex core and the region near the sample edge with the flowing Meissner current, we present in Fig. 6 radial distributions of the heat flow $\dot{Q}_{L}(r)$ and the flow density $\mathcal{P}_{L}(r)$ (10) for two values of the applied magnetic field $H=H_{s 1}, H_{s 2}$ corresponding to the switching between states $L=0 \rightarrow 1$ and $L=1 \rightarrow 2$, respectively. In the Meissner state the spatially resolved heat flow density $\mathcal{P}_{L}(r)$ and the heat flow $\dot{Q}_{L}(r)$ are more pronounced near the disk edge where the screening SC currents have higher density and the SC order parameter is suppressed; Fig. 6(a). A vortex in the mesoscopic SC disk leads to QP redistribution so that the heat flow density in the vortex core is always higher than near the disk edge $\left[\mathcal{P}_{L \neq 0}(0)>\mathcal{P}_{L \neq 0}(R)\right]$. Despite this, the contribution of the region outside the vortex core to the total e-ph heat flow appears to be comparable to the contribution of the vortex core itself because of the relatively small volume of the core region. Note that the significant growth of $\dot{Q}_{L}(r)(10)$ in Figs. 6(b)-6(d) outside the vortex core region illustrates the existence of a noticeable interplay between the contributions from the subgap states, located in the vortex cores and in the region with the reduced spectral gap $E_{\mathrm{g}}$ near the sample edge. The

interplay increases when the field is swept from $H_{S L}$ to $H_{s(L+1)}$ for a fixed vorticity $L$ and with the increase in the number of vortices $L$ trapped in the center of the sample.

The electronic properties of the vortex states will be surely modified if we further increase the radius of the disk $R$ compared to the coherence length $\xi_{0}$. In this case the core of a multiquantum vortex does not extend to the edge of the disk, and QPs in the vortex core remain well localized near the disk center. Clearly in this case the profiles of the heat flow density $\mathcal{P}_{L}(r)$ and the heat flow $\dot{Q}_{L}(r)(10)$ have to reveal a local minimum and a plateau, respectively.

\section{SEMIQUANTITATIVE HOMOGENEOUS APPROXIMATION FOR THE E-PH HEAT FLOW}

For the analysis of the experimental data it is often useful and convenient to build some semiquantitative approximations for the measurable quantities which would allow to avoid the extensive use of the numerical simulations. To develop such a description we try to fit the above numerical simulations of the magnetic field dependence of the e-ph heat flow $\dot{Q}_{L}(H)$ (see Fig. 5) by a simplified model taking into account that the overall spectral characteristics and LDOSs of the mesoscopic sample result from the interplay of the subgap states, located in the vortex core and in the edge regions with the spectral gap reduced by Meissner currents [69]. We assume that both the order parameter $\Delta_{L}(r)$ and the $\theta_{L}(r)$ function vanish inside the vortex core and separate the energy and the coordinate dependence in $\operatorname{LDOS} N_{L}(r, E)$ and SC correlations $B_{L}(r, E)(12)$ by the following approximate expressions (see Fig. 7),

$$
\begin{aligned}
& N_{L}(r, E) \simeq\left[1-Z_{\mathcal{D}_{L}}(r)\right] \bar{N}_{L}(E)+Z_{\mathcal{D}_{L}}(r) \bar{N}_{V}(E), \\
& B_{L}(r, E) \simeq\left[1-Z_{\mathcal{D}_{L}}(r)\right] \bar{B}_{L}(E)+Z_{\mathcal{D}_{L}}(r) \bar{B}_{V}(E) .
\end{aligned}
$$

Here the functions $\bar{N}_{V}(E)=\operatorname{Re}\left[\cos \theta_{V}\right]=1$ and $\bar{B}_{V}(E)=$ $\operatorname{Im}\left[\sin \theta_{V}\right]=0\left(\theta_{V}=0\right)$ describe normal metal properties of the vortex core region. A radial profile $Z_{\mathcal{D}_{L}}(r)$ which describes a contribution of the subgap states in the vortex core $\left(Z_{\mathcal{D}_{L}}(0)=1, Z_{\mathcal{D}_{L}}(R) \ll 1\right)$ is assumed to be a monotonically 


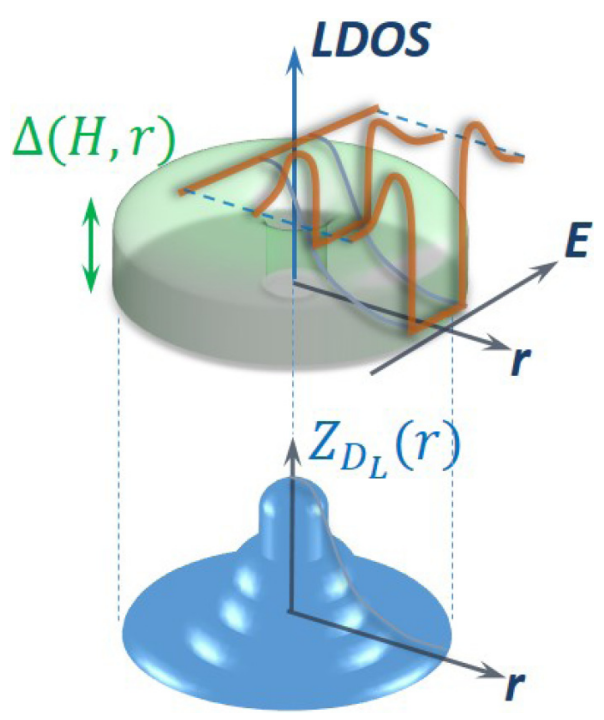

FIG. 7. Sketch of the homogeneous approximation. (Top) The soft SC gap (shown by semitransparent green color) in the SC disk of the radius $R$ with the giant $L$-quantum vortex is accompanied by the energy dependence of the LDOSs (orange lines), Eq. (14), being a superposition of the vortex $\bar{N}_{V}(E)=1$ and homogeneous $\bar{N}_{L}(E)$ LDOS with the radial profile $Z_{\mathcal{D}_{L}}(r)$ (shown by two parallel blue curves). (Bottom) The cylindrical-symmetric representation of the radial function $Z_{\mathcal{D}_{L}}(r)$ in the SC disk.

decaying function with a certain $L$-dependent length scale $\mathcal{D}_{L}$; see Fig. 7 (bottom). A residual small contribution of the subgap states related to the vortex core at the disk edge $\left[\sim Z_{\mathcal{D}_{L}}(R)\right]$ corresponds to a nonzero DOS at the Fermi level and is responsible for the destruction of the hard gap in the DOS. In the Meissner state we get $Z_{0}(r)=0$ and thus $\mathcal{D}_{0}=0$.

The functions $\bar{N}_{L}(E)$ and $\bar{B}_{L}(E)$ describe the averaged characteristics of the SC state in the vortex free phase and outside the vortex core region. To get these functions we average Eq. (3) over the radial coordinate neglecting the gradient terms, i.e., assume the $\theta_{L}(r)$ function to depend slowly on $r$. The spatially averaged value $\bar{\theta}_{L}$ satisfies the Usadel equation for the normal $\left(\cos \bar{\theta}_{L}\right)$ and anomalous $\left(-i \sin \bar{\theta}_{L}\right)$ Green's functions [75]

$$
\left(i E-\bar{\Gamma}_{L} \cos \bar{\theta}_{L}\right) \sin \bar{\theta}_{L}=\bar{\Delta}_{L} \cos \bar{\theta}_{L},
$$

which describes different depairing effects in dirty SCs with the effective depairing energy $\bar{\Gamma}_{L}$ [71,76-78]. The solution $\bar{\theta}_{L}$ of the algebraic equation (16) at $\omega_{n}=-i E$ gives us the energy dependence of the DOS $\bar{N}_{L}(E)=\operatorname{Re}\left[\cos \bar{\theta}_{L}\right]$ and the function $\bar{B}_{L}(E)=\operatorname{Im}\left[\sin \bar{\theta}_{L}\right]$. The hard gap $\bar{E}_{\mathrm{g}}$ in the DOSs $\bar{N}_{L}(E)$ and the order parameter $\bar{\Delta}_{L}$ are determined by the relations

$$
\bar{E}_{\mathrm{g}}=\bar{\Delta}_{L}\left(1-\gamma_{L}^{2 / 3}\right)^{3 / 2}, \quad \bar{\Delta}_{L}=\Delta_{0} \mathrm{e}^{-\pi \gamma_{L} / 4},
$$

which in turn depend on the magnetic field $H$ via parameter

$$
\gamma_{L}=\bar{\Gamma}_{L} / \bar{\Delta}_{L}
$$

The effective depairing energy $\bar{\Gamma}_{L}$ takes into account the phase gradient created by the $L$-quantum vortex and the effect of the magnetic field $H$ and can be calculated by averaging the inhomogeneous depairing parameter $\Gamma_{L}(r, H)(4),(5)$ :

$$
\begin{aligned}
\bar{\Gamma}_{L}\left(H, \mathcal{D}_{L}\right) & =\frac{\pi \hbar}{D S_{Z}} \int_{0}^{R} r\left[1-Z_{\mathcal{D}_{L}}(r)\right]^{2} v_{L}^{2}(r) d r, \\
S_{Z} & =2 \pi \int_{0}^{R} r\left[1-Z_{\mathcal{D}_{L}}(r)\right] d r .
\end{aligned}
$$

The relation (19) accounts for the presence of the core of the $L$-quantum vortex via the factor $1-Z_{\mathcal{D}_{L}}(r)$ in the superfluid velocity $\left[1-Z_{\mathcal{D}_{L}}(r)\right] v_{L}(r)$ and the effective area of the disk $S_{Z}$. As a result, the effective depairing energy $\bar{\Gamma}_{L}(19)$ and parameter $\gamma_{L}$ (18) depend on the magnetic field $H$ and the length scale $\mathcal{D}_{L}$. The kernel (11) takes the form

$$
\begin{aligned}
M_{L}(r, E, \epsilon) \simeq & Z_{\mathcal{D}_{L}}(r)^{2}+\left[1-Z_{\mathcal{D}_{L}}(r)\right]^{2} \bar{M}_{L}(E, \epsilon)+ \\
& +Z_{\mathcal{D}_{L}}(r)\left[1-Z_{\mathcal{D}_{L}}(r)\right] \bar{K}_{L}(E, \epsilon),
\end{aligned}
$$

where the functions

$$
\begin{gathered}
\bar{M}_{L}(E, \epsilon)=\bar{N}_{L}(E) \bar{N}_{L}(E+\epsilon)-\bar{B}_{L}(E) \bar{B}_{L}(E+\epsilon), \\
\bar{K}_{L}(E, \epsilon)=\bar{N}_{L}(E)+\bar{N}_{L}(E+\epsilon)
\end{gathered}
$$

do not depend on radius $r$.

In the Meissner state $\left(L=0, Z_{0}=0\right)$ the depairing energy $\bar{\Gamma}_{0}$ can be easily calculated by the averaging of the inhomogeneous depairing parameter $\Gamma_{0}(r, H)(4),(5)$ over the radial direction

$$
\bar{\Gamma}_{0}(H)=\frac{2}{R^{2}} \int_{0}^{R} d r r \Gamma_{0}(r, H)=\frac{\hbar D}{4 R^{2}}\left(\frac{H}{H_{0}}\right)^{2} .
$$

The kernel $M_{0}(r, E, \epsilon)$ of the second integral in (10) does not depend on radial coordinate $r\left(M_{0}(r, E, \epsilon) \simeq \bar{M}_{0}(E, \epsilon)\right)$ and can be found by replacing the functions $N_{0}(r, E)$ and $B_{0}(r, E)$ with $\bar{N}_{0}(E)$ and $\bar{B}_{0}(E)$, respectively, in the expression (11). The comparison of the above averaged description with the full numerical solution for the DOSs can be seen in Fig. 4(e) where the homogeneous DOSs $\bar{N}_{0}(E)=\operatorname{Re}\left[\cos \bar{\theta}_{0}\right]$ determined by the solution of Eq. (16) is shown.

In the vortex state $(L \neq 0)$ the unknown scale $\mathcal{D}_{L}$ depends on the disk geometry via the spatial distributions of the order parameter $\Delta_{L}(r)$ and the LDOSs $N_{L}(r, E)$ in the disk, and in general $\mathcal{D}_{L}$ is a function of temperature $T$, magnetic field $H$ and vorticity $L$. In order to account for the reduction in the average order parameter $\bar{\Delta}_{L}$ and the minigap $\bar{E}_{\mathrm{g}}$ which occurs with increasing $H$ for a fixed vorticity $L$, the cutoff radius $\mathcal{D}_{L}$ is assumed to obey the following relation

$$
\mathcal{D}_{L}(H)=a_{L} \xi_{L}(H),
$$

where the coherence length

$$
\xi_{L}(H)=\sqrt{\hbar D / 2 \bar{E}_{\mathrm{g}}}
$$

plays the role of the characteristic length scale of the Green's functions outside the vortex core, and $a_{L}$ is a fixed fitting parameter for the $L-$ th orbital mode.

Finally, the expressions in Eqs. (17)-(19), (24), and (25) define the implicit relation between the scale $\mathcal{D}_{L}$ and magnetic field $H$, which after the substitution into the expression (19) for the homogeneous depairing energy $\bar{\Gamma}_{L}$ gives us the average normal $\left(\sim \cos \bar{\theta}_{L}\right)$ and anomalous 
$\left(\sim \sin \bar{\theta}_{L}\right)$ Green's functions from the solution of the algebraic equation (16).

The general expressions (10) for the electron-phonon heat flow can be significantly simplified in low temperature limit $T_{\mathrm{ph}} \ll T \ll \bar{E}_{g} / k_{B}$, where we neglect the terms $e^{-\bar{E}_{g} / k_{B} T_{\mathrm{ph}}}$ with respect to $e^{-\bar{E}_{g} / k_{B} T}$, using approximation of the kernel of the integrals $M(r, E, \epsilon)(20)-(22)$. We calculate the coordinateresolved electron-phonon heat flux $\mathcal{P}_{L}(r)$ for any profile $Z_{\mathcal{D}_{L}}(r)$ combining the procedure, described in Refs. [38,40], and the solution of Usadel equation (16) (see the Appendix for details).

As a result in the leading approximation in $\bar{E}_{g} /\left(k_{B} T\right)$ for $T_{\mathrm{ph}}=0$ and $T \ll \bar{\Gamma}^{2 / 3} \bar{E}_{g}^{1 / 3} / k_{B}$ the electron-phonon heat flow $\dot{Q}_{L}(r)$ within the central part of the disk of the a radius $r \leqslant R$ into the phonon bath for the orbital mode $L$ is given by the expressions

$$
\dot{Q}_{L}(r)=\frac{\dot{Q}_{N}}{\pi R^{2}} \int_{0}^{r} d r^{\prime} r^{\prime} \overline{\mathcal{P}}_{L}\left(r^{\prime}\right),
$$

$$
\begin{aligned}
\overline{\mathcal{P}}_{L}(r) \approx & 2 \pi\left\{Z_{\mathcal{D}_{L}}^{2}(r)+\left[1-Z_{\mathcal{D}_{L}}(r)\right]^{2} \frac{128}{189 \zeta(5)} \frac{k_{B} T}{\Gamma^{2 / 3} \bar{E}_{g}^{1 / 3}}\left[1+\frac{21 \pi}{256}\left(\frac{\bar{E}_{g}}{k_{B} T}\right)^{3} \mathrm{e}^{-\bar{E}_{g} / k_{B} T}\right] \mathrm{e}^{-\bar{E}_{g} / k_{B} T}+\right. \\
& \left.+Z_{\mathcal{D}_{L}}(r)\left[1-Z_{\mathcal{D}_{L}}(r)\right] \frac{\sqrt{\pi / 6}}{48 \zeta(5)}\left(\frac{\bar{E}_{g} \bar{\Delta}}{\bar{\Gamma}^{2}}\right)^{1 / 3}\left(\frac{\bar{E}_{g}}{k_{B} T}\right)^{7 / 2} \mathrm{e}^{-\bar{E}_{g} / k_{B} T}\right\},
\end{aligned}
$$

where the first term in curvy brackets in (27) corresponds to the normal core contribution, the second term - to the SC part with the hard gap, while the last term provides the crosscontribution with the kernel (22). $Q_{N}=\mathcal{V} \Sigma T^{5}$ is the e-ph heat flow in the normal state of the disk of volume $\mathcal{V}=\pi R^{2} d$.

The results of the fitting for the trial function $Z_{\mathcal{D}_{L}}(r)=$ $\mathrm{e}^{-r^{2} / \mathcal{D}_{L}^{2}}$ are shown in Fig. 5. The best fits of $\dot{Q}_{L}(R)(26)$, (27) to the numerical simulations are obtained with $a_{L} \simeq$ $1.5 ; 2.5 ; 4.1 ; 8.0$ for $L=1 \div 4$, respectively. The inset in Fig. 5 shows the dependence of the value $\bar{\Gamma}^{2 / 3} \bar{E}_{g}^{1 / 3}$ (solid lines) and the hard gap $\bar{E}_{g}$ (dashed lines) determined by the relations (17) on the magnetic flux $\phi$ for the best fits. The condition $\bar{\Gamma}^{2 / 3} \bar{E}_{g}^{1 / 3}>k_{B} T$ determines the values of the applied magnetic field $H \gtrsim H_{0}$ for which the approximate expressions (26), (27) are correct. One can see from Fig. 5 that within the validity range $\bar{\Gamma}^{2 / 3} \bar{E}_{g}^{1 / 3}>k_{B} T$ the approximation agrees reasonably well with the exact result. In the range $H<H_{0}$ of the Meissner regime, where the above validity condition is violated, our semiquantitative model obviously fails as it does not take into account highly inhomogeneous distribution of Meissner currents [see, e.g., $P_{0}$ in Fig. 6(a)].

Figures 4(e)-4(h) provide the comparison of the exact spatially resolved LDOS $N_{L}(r, E)$ vs energy $E$ (12) shown in Figs. 4(a)-4(d) to the approximate ones (14) corresponding to the best fits for values of the magnetic field $H=H_{s 1}, H_{s 2}$.

The corresponding spatially resolved heat flow density $\overline{\mathcal{P}}_{L}(r)(27)$ and the heat flow $\dot{Q}_{L}(r)(26)$ are shown in Fig. 6 by the dashed lines. One should notice the semiquantitative agreement between exact numerically calculated and approximate curves, except for the cases Figs. 6(a) and 6(c), when the Meissner screening currents essentially suppress the SC order parameter $\Delta(r)$ near the disk edge, because the simple approximate model (14), (15) fails to account for this suppression.

Figure 8 shows the dependencies of the coherence length $\xi_{L}(H)$ and the corresponding length scale $\mathcal{D}_{L}(H)$ on the magnetic field $H$ for different vorticities $L$. For a fixed vorticity $L$ the coherence length $\xi_{L}(H)$ (25) grows while sweeping the magnetic field up, $H_{S L} \leqslant H \leqslant H_{S(L+1)}$, due to increase of the screening currents in a small disk and shrinking of the gap $\bar{E}_{\mathrm{g}}$. As a result, the radius $\mathcal{D}_{L}$ increases slightly within the branch $L$. The downward jumps in the coherence length $\xi_{L}(H)$ (25) at $H=H_{s L}$ are caused by the increase in the hard spectral gap value $\bar{E}_{\mathrm{g}}$ as the vortex enters the sample, see Fig. 4.

\section{CONCLUSIONS}

In conclusion, on the basis of the Usadel theory we have calculated the e-ph heat transfer $\dot{Q}$ in a diffusive mesoscopic SC disk of the size comparable to several SC coherence lengths $\xi_{0}$, placed in the external magnetic field $H$ oriented perpendicular to the plane of the disk. The strong confinement effects of the screening supercurrents are responsible for the formation of the giant ( $L$-quantum) vortices in the disk center. The giant vortex core and the regions near the

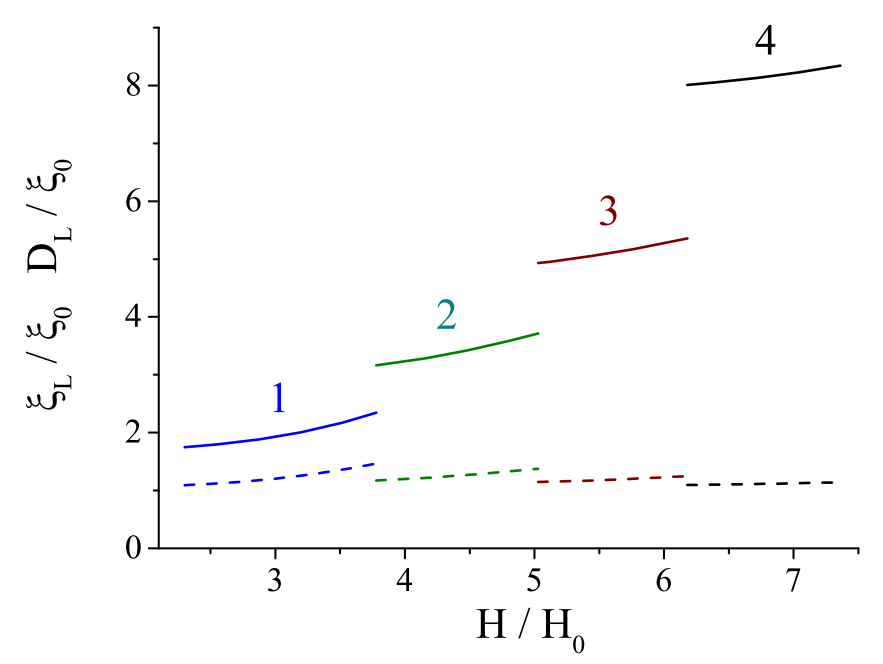

FIG. 8. The dependence of the scale $\mathcal{D}_{L}$ (solid lines) and the coherence length $\xi_{L}$ (dashed lines) on the magnetic field $H$ in the SC disk of the radius $R=4 \xi_{0}$ for the temperature $T=0.1 T_{\mathrm{cs}}$. The numbers near the curves denote the corresponding values of vorticity $L$. 
sample edge with the reduced spectral gap form potential wells (traps) for QPs responsible for the heat transfer. We have demonstrated that the transitions between the SC states with different vorticities $L$ provoke abrupt changes (jumps) in $\dot{Q}(H)$ attributed to the entry/exit of vortices while sweeping the magnetic field. The smooth growth of the e-ph heat flow $\dot{Q}_{L}(H)$ takes place for a fixed vorticity $L$ while sweeping the magnetic field up, due to the increase of the screening currents and shrinking of the hard or soft spectral gap in the density of states. We have shown that the e-ph heat flow in the Meissner and vortex states of mesoscopic samples can be effectively controlled by the external magnetic field. We develop the semiquantitative approximate model the description of the QP trapping which works reasonably well beyond the Meissner state. Our numerical analysis of the e-ph relaxation confirms the validity and efficiency of the above semiquantitative model. Our semiquantitative approach provides an access to more detailed characteristics like a spatially-resolved electron-phonon relaxation rate (see Fig. 6) and a value of the soft SC gap $E_{g}$ (see Fig. 5) which cannot be directly accessed in the experiments. The information about these more detailed characteristics allows experimentalists to design the tunable and efficient QP traps in order to optimize the performance of their quantum SC devices. Our consideration is restricted to the case of giant vortices and does not include the analysis of possible consequences of the giant vortex decay and formation of the vortex molecules. One can obviously expect that for rather small splitting of a giant vortex, i.e. for the distances between the individual vortex centers smaller than the giant vortex core size, our results for the electron-phonon heat transfer are still applicable. The heat transfer in the general case of the strongly split vortex molecules deserves certainly a separate consideration which goes beyond the perspective of the current manuscript. We expect that our results can stimulate further experimental work on the controllable QPs trapping in the vortex state of mesoscopic SCs and SC hybrid devices.

\section{ACKNOWLEDGMENTS}

We acknowledge Prof. Jukka P. Pekola for fruitful discussions. This work was supported, by the Center of Excellence "Center of Photonics" funded by The Ministry of Science and Higher Education of the Russian Federation, contract 075-152020-906.

\section{APPENDIX: HOMOGENEOUS APPROXIMATION IN A MESOSCOPIC DISK}

In this section we derive the expression for the e-ph heat flow $\dot{Q}_{\text {eph }}[(10)-(12)]$ in a mesoscopic SC disk with a $L$ quantum vortex in the center within the approximation (14) and (15). Using the kernel (20), we decompose the heat flow density (10) into three parts as follows,

$$
\mathcal{P}_{L}(r)=\Pi^{(1)} F^{2}(r)+\Pi^{(2)}[1-F(r)]^{2}+\Pi^{(3)} F(r)[1-F(r)],
$$

where the coefficients $\Pi^{(i)}$ are given by the unified expression

$$
\Pi_{L}^{(i)}=\frac{\Sigma}{24 \zeta(5) k_{B}^{5}} \int_{0}^{\infty} \epsilon^{3}\left[n_{T}(\epsilon)-n_{T_{\mathrm{ph}}}(\epsilon)\right] d \epsilon \int_{-\infty}^{\infty} \mathcal{M}_{L}^{(i)}(E, \epsilon)\left[f_{T}(E)-f_{T}(E+\epsilon)\right] d E,
$$

with the kernels $\mathcal{M}_{L}^{(1)}(E, \epsilon)=1, \mathcal{M}_{L}^{(2)}(E, \epsilon)=\bar{M}_{L}(E, \epsilon)$, and $\mathcal{M}_{L}^{(3)}(E, \epsilon)=\bar{K}_{L}(E, \epsilon)$, respectively. The coefficient

$$
\Pi^{(1)}=\frac{\Sigma}{24 \zeta(5) k_{B}^{5}} \int_{0}^{\infty} \epsilon^{3}\left[n_{T}(\epsilon)-n_{T_{\mathrm{ph}}}(\epsilon)\right] d \epsilon \int_{-\infty}^{\infty}\left[f_{T}(E)-f_{T}(E+\epsilon)\right] d E=\Sigma\left[T^{5}-T_{\mathrm{ph}}^{5}\right],
$$

describes the heat flow density in the normal state and determines the contribution of the vortex core.

For simplicity, further calculations are done in low-temperature limit $T_{0} \ll T \ll \bar{E}_{g} / k_{B}$ in order to neglect the temperature dependence of the gap and the order parameter and the terms $e^{-\bar{E}_{g} / k_{B} T_{\mathrm{ph}}}$ with respect to $e^{-\bar{E}_{g} / k_{B} T}$. In this case, the values $\Pi^{(i)}$ (A2) do not depend on the phonon temperature $T_{\mathrm{ph}}$ and $\Pi^{(1)} \approx \Sigma T^{5}$.

In the low-temperature limit the main contributions to the coefficients $\Pi^{(2)}$ and $\Pi^{(3)}$ arise from the vicinity $0<|E|-\bar{E}_{g} \lesssim$ $k_{B} T$ of the hard gap value $\pm \bar{E}_{g}$, leading to the smallness of the positive parameter $\delta E=|E|-\bar{E}_{g} \ll \bar{E}_{g}$. The expansion of $\bar{N}_{L}(E)$ and $\bar{B}_{L}(E)$ over $\delta E$ takes the form

$$
\bar{N}_{L}^{2}(E) \simeq \Theta(\delta E) \frac{2 \delta E \bar{\Delta}_{L}^{2 / 3}}{3 \bar{\Gamma}_{L}^{4 / 3} \bar{E}_{g}^{1 / 3}}, \quad \frac{\bar{B}_{L}(E)}{\bar{N}_{L}(E) \operatorname{sign}(E)} \simeq\left(\frac{\bar{E}_{g}}{\bar{\Delta}_{L}}\right)^{1 / 3}
$$

Here $\Theta(x)$ is the Heaviside theta function. Substituting (A4) into expressions (21) and (22) we obtain the final expression for the kernels $\bar{M}_{L}(E, \epsilon)$ and $\bar{K}_{L}(E, \epsilon)$

$$
\bar{M}_{L}(E, \epsilon) \simeq \frac{2 \sqrt{\delta E \delta E^{\prime}} \Theta(\delta E) \Theta\left(\delta E^{\prime}\right)}{3 \bar{\Gamma}_{L}^{2 / 3} \bar{E}_{g}^{1 / 3}}, \quad \bar{K}_{L}(E, \epsilon) \simeq \sqrt{\frac{2 \bar{\Delta}_{L}^{2 / 3}}{3 \bar{\Gamma}_{L}^{4 / 3} \bar{E}_{g}^{1 / 3}}}\left[\sqrt{\delta E} \Theta(\delta E)+\sqrt{\delta E^{\prime}} \Theta\left(\delta E^{\prime}\right)\right],
$$

which are correct for $T \ll \bar{\Gamma}^{2 / 3} \bar{E}_{g}^{1 / 3} / k_{B}$, where $\delta E^{\prime}=|E+\epsilon|-\bar{E}_{g}$. Splitting the integration in $\Pi^{(2)}$ (A2) into positive and negative parts in the electronic energy $E$ and taking into account that $\bar{M}(E, \epsilon)=0$ if any of two conditions $|E|<\bar{E}_{g}$ and 
$|E+\epsilon|<\bar{E}_{g}$ is valid, the coefficient $\Pi^{(2)}$ can be simplified in the low-temperature limit as follows,

$$
\Pi^{(2)} \approx \frac{\Sigma}{24 \zeta(5) k_{B}^{5}}\left[2 \int_{0}^{\infty} \epsilon^{3} \mathrm{e}^{-\epsilon / k_{B} T} d \epsilon \int_{\bar{E}_{g}}^{\infty} \bar{M}_{L}(E, \epsilon) \mathrm{e}^{-E / k_{B} T} d E+\int_{2 \bar{E}_{g}}^{\infty} \epsilon^{3} \mathrm{e}^{-\epsilon / k_{B} T} d \epsilon \int_{\bar{E}_{g}-\epsilon}^{-\bar{E}_{g}} \bar{M}_{L}(E, \epsilon) d E\right] .
$$

As a result, taking into account only the leading terms in the small parameter $k_{B} T / \bar{E}_{g}$, we obtain

$$
\Pi^{(2)}=\frac{128 \Sigma T^{5}}{189 \zeta(5)} \frac{k_{B} T}{\bar{\Gamma}_{L}^{2 / 3} \bar{E}_{q}^{1 / 3}}\left[1+\frac{21 \pi}{256}\left(\frac{\bar{E}_{g}}{k_{B} T}\right)^{3} \mathrm{e}^{-\bar{E}_{g} / k_{B} T}\right] \mathrm{e}^{-\bar{E}_{g} / k_{B} T}
$$

In order to simplify the expression for $\Pi^{(3)}$ with $E \geqslant \bar{E}_{g}$, we neglect $n_{T_{\mathrm{ph}}}(\epsilon)$ and use the following equalities,

$$
n_{T}(\epsilon) f_{T}(E-\epsilon)=f_{T}(E)\left[n_{T}(\epsilon)+f_{T}(E-\epsilon)\right], \quad n_{T}(\epsilon) f_{T}(E+\epsilon)=f_{T}(E)\left[n_{T}(\epsilon)-f_{T}(E+\epsilon) \mathrm{e}^{E / k_{B} T}\right]
$$

As a result, we obtain

$$
\Pi^{(3)} \approx \frac{\Sigma}{12 \zeta(5) k_{B}^{5}} \int_{0}^{\infty} \epsilon^{3} n_{T}(\epsilon) d \epsilon \int_{\bar{E}_{g}}^{\infty} \bar{N}_{L}(E)\left[f_{T}(E-\epsilon)+f_{T}(E+\epsilon) \mathrm{e}^{E / k_{B} T}\right]
$$

leading to

$$
\Pi^{(3)} \approx \frac{\sqrt{\pi / 6} \Sigma T^{5}}{48 \zeta(5)}\left(\frac{\bar{E}_{g} \bar{\Delta}_{L}}{\bar{\Gamma}_{L}^{2}}\right)^{1 / 3}\left(\frac{\bar{E}_{g}}{k_{B} \bar{T}}\right)^{7 / 2} \mathrm{e}^{-\bar{E}_{g} / k_{B} \bar{T}}
$$

Finally, substituting Eqs. (A1), (A3), (A8), (A9) into the expressions (10), one obtains simplified expressions (26), (27) for the electron-phonon heat flow $\dot{Q}_{L}(r)$ into the phonon bath for the orbital mode $L$.

[1] F. Giazotto, T. T. Heikkila, A. Luukanen, A. M. Savin, and J. P. Pekola, Opportunities for mesoscopics in thermometry and refrigeration: Physics and applications, Rev. Mod. Phys. 78, 217 (2006).

[2] A. Shurakov, Y. Lobanov, and G. Goltsman, Superconducting hot-electron bolometer: From the discovery of hot-electron phenomena to practical applications, Supercond. Sci. Technol. 29, 023001 (2016).

[3] A. D. Semenov, G. N. Goltsman, and R. Sobolewski, Hot-electron effect in superconductors and its applications for radiation sensors, Supercond. Sci. Technol. 15, R1 (2002).

[4] M. Kjaergaard, M. E. Schwartz, J. Braumüller, P. Krantz, J. I.-J. Wang, S. Gustavsson, and W. D. Oliver, Superconducting qubits: Current state of play, Annu. Rev. Condens. Matter Phys. 11, 369 (2020).

[5] M. H. Devoret, A. Wallraff, and J. M. Martinis, Superconducting qubits: A short review, arXiv:cond-mat/0411174.

[6] G. Catelani and J. P. Pekola, Using materials for quasiparticle engineering, arXiv:2107.09695, Mater. Quantum Technol. (2022), doi: 10.1088/2633-4356/ac4a75.

[7] J. M. Martinis, M. Ansmann, and J. Aumentado, Energy Decay in Superconducting Josephson-Junction Qubits from Nonequilibrium Quasiparticle Excitations, Phys. Rev. Lett. 103, 097002 (2009).

[8] H. Paik, D. I. Schuster, L. S. Bishop, G. Kirchmair, G. Catelani, A. P. Sears, B. R. Johnson, M. J. Reagor, L. Frunzio, L. I. Glazman, S. M. Girvin, M. H. Devoret, and R. J. Schoelkopf, Observation of High Coherence in Josephson Junction Qubits Measured in a Three-Dimensional Circuit QED Architecture, Phys. Rev. Lett. 107, 240501 (2011).
[9] A. D. Córcoles, J. M. Chow, J. M. Gambetta, C. Rigetti, J. R. Rozen, G. A. Keefe, M. B. Rothwell, M. B. Ketchen, and M. Steffen, Protecting superconducting qubits from radiation, Appl. Phys. Lett. 99, 181906 (2011).

[10] H. Wang, M. Hofheinz, J. Wenner, M. Ansmann, R. C. Bialczak, M. Lenander, E. Lucero, M. Neeley, A. D. O'Connell, D. Sank, M. Weides, A. N. Cleland, and J. M. Martinis, Improving the Coherence Time of Superconducting Coplanar Resonators, Appl. Phys. Lett. 95, 233508 (2009).

[11] R. Barends, J. Wenner, M. Lenander, Y. Chen, R. C. Bialczak, J. Kelly, E. Lucero, P. O’Malley, M. Mariantoni, D. Sank, H. Wang, T. C. White, Y. Yin, J. Zhao, A. N. Cleland, J. M. Martinis, and J. J. A. Baselmans, Minimizing Quasiparticle Generation from stray Infrared light in Superconducting Quantum Circuits, Appl. Phys. Lett. 99, 113507 (2011).

[12] J. P. Pekola, D. V. Anghel, T. I. Suppula, J. K. Suoknuuti, A. J. Manninen, and M. Manninen, Trapping of quasiparticles of a nonequilibrium superconductor, Appl. Phys. Lett. 76, 2782 (2000).

[13] S. Rajauria, H. Courtois, and B. Pannetier, Quasiparticlediffusion-based heating in superconductor tunneling microcoolers, Phys. Rev. B 80, 214521 (2009).

[14] H. S. Knowles, V. F. Maisi, and J. P. Pekola, Probing quasiparticle excitations in a hybrid single electron transistor, Appl. Phys. Lett. 100, 262601 (2012).

[15] D. M. T. van Zanten, D. M. Basko, I. M. Khaymovich, J. P. Pekola, H. Courtois, and C. B. Winkelmann, Single Quantum Level Electron Turnstile, Phys. Rev. Lett. 116, 166801 (2016).

[16] I. M. Khaymovich and D. M. Basko, Recovery of a SINIS turnstile accuracy in a strongly non-equilibrium regime, Phys. Rev. B 94, 165158 (2016). 
[17] M. Marín-Suárez, J. T. Peltonen, D. S. Golubev, and J. P. Pekola, Frequency to power conversion by an electron turnstile, Nat. Nanotechnol. (2022), doi: 10.1038/s41565-021-01053-5.

[18] P. J. de Visser, J. J. A. Baselmans, P. Diener, S. J. C. Yates, A. Endo, and T. M. Klapwijk, Generation-recombination noise: The fundamental sensitivity limit for kinetic inductance detectors, J. Low Temp. Phys. 167, 335 (2012).

[19] K. Yu. Arutyunov, S. A. Chernyaev, T. Karabassov, D. S. Lvov, V. S. Stolyarov, and A. S. Vasenko, Relaxation of nonequilibrium quasiparticles in mesoscopic size superconductors, J. Phys.: Condens. Matter 30, 343001 (2018).

[20] D. J. Goldie, N. E. Booth, C. Patel, and G. L. Salmon, Quasiparticle Trapping from a Single-Crystal Superconductor into a Normal-Metal Film Via the Proximity Effect, Phys. Rev. Lett. 64, 954 (1990).

[21] J. N. Ullom, P. A. Fisher, and M. Nahum, Measurements of quasiparticle thermalization in a normal metal, Phys. Rev. B 61, 14839 (2000).

[22] S. Rajauria, L. M. A. Pascal, P. Gandit, F. W. J. Hekking, B. Pannetier, and H. Courtois, Efficiency of quasiparticle evacuation in superconducting devices, Phys. Rev. B 85, 020505(R) (2012).

[23] E. M. Levenson-Falk, F. Kos, R. Vijay, L. Glazman, and I. Siddiqi, Single Quasiparticle Trapping in Aluminum Nanobridge Josephson Junctions, Phys. Rev. Lett. 112, 047002 (2014).

[24] A. A. Golubov, E. P. Houwman, J. G. Gijsbertsen, J. Flokstra, H. Rogalla, J. B. le Grand, and P. A. J. de Korte, Quasiparticle lifetimes and tunneling times in a superconductor-insulatorsuperconductor tunnel junction with spatially inhomogeneous electrodes, Phys. Rev. B 49, 12953 (1994).

[25] S. Friedrich, K. Segall, M. C. Gaidis, C. M. Wilson, and D. E. Prober, Experimental quasiparticle dynamics in a superconducting, imaging x-ray spectrometer, Appl. Phys. Lett. 71, 3901 (1997).

[26] J. Aumentado, M. W. Keller, and J. M. Martinis, and M. H. Devoret, Nonequilibrium Quasiparticles and 2e Periodicity in Single-Cooper-Pair Transistors, Phys. Rev. Lett. 92, 066802 (2004).

[27] N. A. Court, A. J. Ferguson, and R. Lutchyn, and R. G. Clark, Quantitative study of quasiparticle traps using the singleCooper-pair transistor, Phys. Rev. B 77, 100501(R) (2008).

[28] J. T. Peltonen, J. T. Muhonen, M. Meschke, N. B. Kopnin, and J. P. Pekola, Magnetic-field-induced stabilization of nonequilibrium superconductivity in a normalmetal/insulator/superconductor junction, Phys. Rev. B 84, 220502(R) (2011).

[29] I. Nsanzineza and B. L. T. Plourde, Trapping a Single Vortex and Reducing Quasiparticles in a Superconducting Resonator, Phys. Rev. Lett. 113, 117002 (2014).

[30] C. Wang, Y. Y. Gao, I. M. Pop, U. Vool, C. Axline, T. Brecht, R. W. Heeres, L. Frunzio, M. H. Devoret, G. Catelani, L. I. Glazman, and R. J. Schoelkopf, Measurement and control of quasiparticle dynamics in a superconducting qubit, Nat. Commun. 5, 5836 (2014).

[31] D. J. van Woerkom, A. Geresdi, and Leo P. Kouwenhoven, One minute parity lifetime of a NbTiN Cooper-pair transistor, Nat. Phys. 11, 547 (2015).

[32] U. Vool, I. M. Pop, K. Sliwa, B. Abdo, C. Wang, T. Brecht, Y. Y. Gao, S. Shankar, M. Hatridge, G. Catelani, M. Mirrahimi,
L. Frunzio, R. J. Schoelkopf, L. I. Glazman, and M. H. Devoret, Non-Poissonian Quantum Jumps of a Fluxonium Qubit Due to Quasiparticle Excitations, Phys. Rev. Lett. 113, 247001 (2014).

[33] M. Taupin, I. M. Khaymovich, M. Meschke, A. S. Mel'nikov, and J. P. Pekola, Tunable quasiparticle trapping in Meissner and vortex states of mesoscopic superconductors, Nat. Commun. 7, 10977 (2016).

[34] S. Nakamura, Y. A. Pashkin, M. Taupin, V. F. Maisi, I. M. Khaymovich, A. S. Mel'nikov, J. T. Peltonen, J. P. Pekola, Y. Okazaki, S. Kashiwaya, S. Kawabata, A. S. Vasenko, J.-S. Tsai, and N.-H. Kaneko, Interplay of the Inverse Proximity Effect and Magnetic Field in Out-of-Equilibrium Single-Electron Devices, Phys. Rev. Appl. 7, 054021 (2017).

[35] K. Ueda, Y. Sato, Y. Takeshige, H. Kamata, K. Li, L. Samuelson, H. Xu, S. Matsuo, and S. Tarucha, Quasiparticle trapping at vortices producing Josephson supercurrent enhancement arXiv:2111.01381.

[36] G. M. Eliashberg, Inelastic electron collisions and nonequilibrium stationary states in superconductors, Zh. Eksp. Teor. Fiz. 61, 1254 (1971) [Sov. Phys. JETP 34, 668 (1972)].

[37] S. B. Kaplan, C. C. Chi, D. N. Langenberg, J. J. Chang, S. Jafarey, and D. J. Scalapino, Quasiparticle and phonon lifetimes in superconductors, Phys. Rev. B 14, 4854 (1976).

[38] N. B. Kopnin, Theory of Nonequilibrium Superconductivity, International Series of Monographs on Physics No. 110 (Oxford University Press, New York, 2001).

[39] F. C. Wellstood, C. Urbina, and J. Clarke, Hot-electron effects in metals, Phys. Rev. B 49, 5942 (1994).

[40] A. V. Timofeev, C. P. García, N. B. Kopnin, A. M. Savin, M. Meschke, F. Giazotto, and J. P. Pekola, Recombination-Limited Energy Relaxation in a Bardeen-Cooper-Schrieffer Superconductor, Phys. Rev. Lett. 102, 017003 (2009).

[41] V. F. Maisi, S. V. Lotkhov, A. Kemppinen, A. Heimes, J. T. Muhonen, and J. P. Pekola, Excitation of Single Quasiparticles in a Small Superconducting Al Island Connected to NormalMetal Leads by Tunnel Junctions, Phys. Rev. Lett. 111, 147001 (2013).

[42] F. S. Bergeret, M. Silaev, P. Virtanen, and T. T. Heikkila, Colloquium: Nonequilibrium effects in superconductors with a spin-splitting field, Rev. Mod. Phys. 90, 041001 (2018).

[43] N. B. Kopnin, Vortex dissipation in clean superconductors at ultralow temperatures, Phys. Rev. B 60, 581 (1999).

[44] A. Rothwarf and B. N. Taylor, Measurement of Recombination Lifetimes in Superconductors, Phys. Rev. Lett. 19, 27 (1967).

[45] D. A. Ivanov and M. V. Feigel'man, Phonon relaxation of subgap levels in superconducting quantum point contacts, JETP Lett. 68, 890 (1998); [Pis'ma v ZhETP 68, 847 (1998)].

[46] M. Zgirski, L. Bretheau, Q. Le Masne, H. Pothier, D. Esteve, and C. Urbina, Evidence for Long-Lived Quasiparticles Trapped in Superconducting Point Contacts, Phys. Rev. Lett. 106, 257003 (2011).

[47] D. G. Olivares, A. L. Yeyati, L. Bretheau, C. O. Girit, H. Pothier, and C. Urbina, Dynamics of quasiparticle trapping in Andreev levels, Phys. Rev. B 89, 104504 (2014).

[48] Y. Savich, L. Glazman, and A. Kamenev, Quasiparticle relaxation in superconducting nanostructures, Phys. Rev. B 96, 104510 (2017).

[49] A. K. Geim, I. V. Grigorieva, S. V. Dubonos, J. G. S. Lok, J. C. Maan, A. E. Filippov, and F. M. Peeters, Phase transitions in 
individual sub-micrometre superconductors, Nature (London) 390, 259 (1997).

[50] A. K. Geim, S. V. Dubonos, J. J. Palacios, I. V. Grigorieva, M. Henini, and J. J. Schermer, Fine Structure in Magnetization of Individual Fluxoid States, Phys. Rev. Lett. 85, 1528 (2000)

[51] M. Morelle, J. Bekaert, and V. V. Moshchalkov, Influence of the sample geometry on the vortex matter in superconducting microstructures, Phys. Rev. B 70, 094503 (2004).

[52] T. Nishio, Q. Chen, W. Gillijns, K. De Keyser, K. Vervaeke, and V. V. Moshchalkov, Scanning Hall probe microscopy of vortex patterns in a superconducting microsquare, Phys. Rev. B 77, 012502 (2008).

[53] H. J. Zhao, V. R. Misko, F. M. Peeters, V. Oboznov, S. V. Dubonos, and I. V. Grigorieva, Vortex states in mesoscopic superconducting squares: Formation of vortex shells, Phys. Rev. B 78, 104517 (2008).

[54] N. Kokubo, S. Okayasu, A. Kanda, and B. Shinozaki, Scanning SQUID microscope study of vortex polygons and shells in weak-pinning disks of an amorphous superconducting film, Phys. Rev. B 82, 014501 (2010).

[55] A. Kanda, B. J. Baelus, F. M. Peeters, K. Kadowaki, and Y. Ootuka, Experimental Evidence for Giant Vortex States in a Mesoscopic Superconducting Disk, Phys. Rev. Lett. 93, 257002 (2004)

[56] T. Cren, D. Fokin, F. Debontridder, V. Dubost, and D. Roditchev, Ultimate Vortex Confinement Studied by Scanning Tunneling Spectroscopy, Phys. Rev. Lett. 102, 127005 (2009).

[57] T. Cren, L. Serrier-Garcia, F. Debontridder, and D. Roditchev, Vortex Fusion and Giant Vortex States in Confined Superconducting Condensates, Phys. Rev. Lett. 107, 097202 (2011).

[58] T. Nishio, T. An, A. Nomura, K. Miyachi, T. Eguchi, H. Sakata, S. Lin, N. Hayashi, N. Nakai, M. Machida, and Y. Hasegawa, Superconducting Pb island Nanostructures Studied by Scanning Tunneling Microscopy and Spectroscopy, Phys. Rev. Lett. 101, 167001 (2008).

[59] M. Timmermans, L. Serrier-Garcia, M. Perini, J. Van de Vondel, and V. V. Moshchalkov, Direct observation of condensate and vortex confinement in nanostructured superconductors, Phys. Rev. B 93, 054514 (2016).

[60] H. F. Hess, R. B. Robinson, R. C. Dynes, J. M. Walles, Jr., and J. V. Waszczak, Scanning-Tunneling-Microscope Observation of the Abrikosov Flux Lattice and the Density of States Near and Inside a Fluxoid, Phys. Rev. Lett. 62, 214 (1989).

[61] A. S. Mel'nikov, I. M. Nefedov, D. A. Ryzhov, I. A. Shereshevskii, V. M. Vinokur, and P. P. Vysheslavtsev, Vortex states and magnetization curve of square mesoscopic superconductors, Phys. Rev. B 65, 140503(R) (2002).

[62] Y. Tanaka, A. Hasegawa, and H. Takayanagi, Energy spectrum of the quasiparticle in a quantum dot formed by a superconduct- ing pair potential under a magnetic field, Solid St. Commun. 85, 321 (1993).

[63] S. M. M. Virtanen and M. M. Salomaa, Multiquantum vortices in superconductors: Electronic and scanning tunneling microscopy spectra, Phys. Rev. B 60, 14581 (1999).

[64] K. Tanaka, I. Robel, and B. Janko, Electronic structure of multiquantum giant vortex states in mesoscopic superconducting disks, Proc. Nat. Acad. Sci. USA 99, 5233 (2002).

[65] N. B. Kopnin, A. S. Mel'nikov, V. I. Pozdnyakova, D. A. Ryzhov, I. A. Shereshevskii, and V. M. Vinokur, Giant Oscillations of Energy Levels in Mesoscopic Superconductors, Phys. Rev. Lett. 95, 197002 (2005).

[66] A. S. Mel'nikov, D. A. Ryzhov, and M. A. Silaev, Electronic structure and heat transport of multivortex configurations in mesoscopic superconductors, Phys. Rev. B 78, 064513 (2008).

[67] A. S. Mel'nikov, D. A. Ryzhov, and M. A. Silaev, Local density of states around single vortices and vortex pairs: Effect of boundaries and hybridization of vortex core states, Phys. Rev. B 79, 134521 (2009).

[68] B. Xu, M. V. Milosevic, S-H. Lin, F. M. Peeters, and B. Jankó, Formation of Multiple-Flux-Quantum Vortices in Mesoscopic Superconductors from Simulations of Calorimetric, Magnetic, and Transport Properties, Phys. Rev. Lett. 107, 057002 (2011).

[69] A. V. Samokhvalov, I. A. Shereshevskii, N. K. Vdovicheva, M. Taupin, I. M. Khaymovich, J. P. Pekola, and A. S. Mel'nikov, Electronic structure of a mesoscopic superconducting disk: Quasiparticle tunneling between the giant vortex core and the disk edge, Phys. Rev. B 99, 134512 (2019).

[70] K. D. Usadel, Generalized diffusion equation for superconducting alloys, Phys. Rev. Lett. 25, 507 (1970).

[71] A. Anthore, H. Pothier, and D. Esteve, Density of States in a Superconductor Carrying a Supercurrent, Phys. Rev. Lett. 90, 127001 (2003).

[72] W. A. Little and R. D. Parks, Observation of Quantum Periodicity in the Transition Temperature of a Superconducting Cylinder, Phys. Rev. Lett. 9, 9 (1962).

[73] R. D. Parks and W. A. Little, Fluxoid quantization in a multiplyconnected superconductor, Phys. Rev. 133, A97 (1964).

[74] H. T. Jadallah, J. Rubinstein, and P. Sternberg, Phase Transition Curves for Mesoscopic Superconducting Samples, Phys. Rev. Lett. 82, 2935 (1999).

[75] For a review, see K. Maki, in Superconductivity, edited by R. D. Parks (Marcel Dekker, NewYork, 1969), p. 1035.

[76] S. Skalski, O. Betbeder-Matibet, P. R. Weiss, Properties of superconducting alloys containing paramagnetic impurities, Phys. Rev. 136, A1500 (1964).

[77] K. Maki and P. Fulde, Equivalence of different pair-breaking mechanisms in superconductors, Phys. Rev. 140, A1586 (1965).

[78] P. Fulde, Tunneling density of states for a superconductor carrying a current, Phys. Rev. 137, A783 (1965). 